\title{
Periodic motions in a gravitational central field with a rotating external force
}

\author{
Alessandro Fonda, Rodica Toader and Pedro J. Torres \\ Abstract. We consider a Kepler problem, with an additional rotating \\ external force, and study the existence of periodic solutions when a small \\ perturbative term is introduced. Surprisingly enough, we always get at \\ least one of such solutions. Moreover, if a nonresonance assumption is \\ added, then the existence of a second solution is also proved.
}

\section{Introduction and main result}

In this paper we consider the existence of periodic solutions of a second order differential equation in the plane of the type

$$
\ddot{x}+\gamma \frac{x}{|x|^{3}}-\alpha \frac{e^{i \omega t}}{|x|^{\beta}}=\varepsilon F(t, x, \dot{x} ; \varepsilon) .
$$

Hence, $x(t)$ is in $\mathbb{R}^{2}$, which will be identified, to simplify the notation, with the complex plane $\mathbb{C}$. All constants $\gamma, \alpha, \omega$ and $\varepsilon$ are assumed to be positive, and we take $\beta \in[0,2]$. In particular, $\omega$ is the frequency of the periodic rotating external force, which has period $T=2 \pi / \omega$. Moreover, $\varepsilon$ is assumed to be a small parameter, and $F: \mathbb{R} \times\left(\mathbb{R}^{2} \backslash\{0\}\right) \times \mathbb{R}^{2} \times[0,1] \rightarrow \mathbb{R}^{2}$ is a continuous and $T$-periodic function, locally Lipschitz continuous in its second and third variables.

Notice that, when $\alpha=0$ and $\varepsilon=0$, equation (1) is the classical twobody Kepler problem, for which it is well-known that, for any given period, there is a continuum of periodic orbits. When $\alpha \neq 0$, the additional term $\alpha e^{i \omega t} /|x|^{\beta}$ introduces a rotating component which naturally leads to the study of $T$-periodic solutions. To our knowledge, such a model has not yet been considered in the literature.

Equation (1) belongs to the wide family of systems with singularities, that have been studied without interruption since the very foundation of Celestial Mechanics, by using a variety of mathematical techniques including analytical, geometrical, topological and variational methods. Classically, the involved models are autonomous (i.e., not explicitly depending on time), but more recently there has been an increasing interest on the study of non-autonomous systems. An additional parametric or external forcing term may model the 
influence of anisotropies, or external periodic gravitational fields. A comprehensive revision of the state-of-the-art is virtually impossible, therefore we only quote some examples of the related literature. The use of topological methods in the study of singular systems goes back to Poincaré [26]. More recently, variational methods have been successfully employed in, e.g., [1, 4, 5, 7, 9, 12, 20, 27]. See also the book [2], and the references therein.

Similar problems have already been considered by the authors of this paper. Radially symmetric systems are studied in $[15,16,17]$ by using topological methods. In this case, the analysis is simplified by the fact that the radial coordinate is ruled by a scalar equation. In [19, 29] (see also [18]), fixed-point theorems on compression-expansion of conical sections are applied, leading to periodic solutions that do not rotate around the origin.

The strategy proposed in this paper is different from the previous ones and falls into the family of perturbative methods. Generally speaking, Perturbation Theory aims to find solutions close to known solutions of the unperturbed system. For the importance of Perturbation Theory in natural sciences in general and Celestial Mechanics in particular, one can read the excellent historical reviews $[22,25]$. The perturbed two-body problem is a classical topic (see for instance $[24,28])$. Recent works where perturbative arguments are applied to singular systems are [3, 23]. Concerning the Kepler problem, there seems to be a still growing interest, as shown by the recent papers $[6,11,13,14,21,30]$, just to quote a few.

In the case under consideration, the aim is to identify periodic solutions close to circular ones. As we will see, the inclusion of the additional forcing term $\alpha e^{i \omega t} /|x|^{\beta}$ fixes the period and, if $\varepsilon=0$, only two circular solutions survive. The objective is then to find conditions to locally continue such solutions for $\varepsilon \neq 0$ small enough.

Our main result is as follows.

Theorem 1 For any choice of the positive constants $\gamma, \alpha, \omega$ and of $\beta \in[0,2]$, there is an $\varepsilon_{0}>0$ such that, if $\varepsilon \in\left[0, \varepsilon_{0}\right]$, then equation (1) has at least one T-periodic solution. Moreover, there are at least two T-periodic solutions if the rotating periodic force is such that

$$
\beta \in[0,2[, \quad \text { or } \quad \beta=2 \text { and } \alpha<\gamma,
$$

and

$$
\frac{\omega^{2(2-\beta)}}{\alpha^{3}} \neq \frac{(a(k)+1)^{\beta+1}}{\gamma^{\beta+1} a(k)^{3}},
$$

for every integer $k$ with $|k| \geq 2$, where $a(k)$ is defined by

$$
a(k)= \begin{cases}\frac{(1-\beta) k^{2}-3+\sqrt{(\beta-3)^{2} k^{4}+2(5 \beta-7) k^{2}+9}}{2(2-\beta)} & \text { if } \beta \in[0,2[, \\ \frac{k^{2}\left(k^{2}-1\right)}{k^{2}+3} & \text { if } \beta=2 .\end{cases}
$$


This is surely true if

$$
\gamma\left(\frac{\omega^{2(2-\beta)}}{\alpha^{3}}\right)^{\frac{1}{\beta+1}}>\frac{19}{12}
$$

The proof of Theorem 1 relies on the spectral analysis of the linearization around equilibria of the system written in a synodic framework. This will be done in the next section. Assumption (2) is introduced in order to avoid some kind of resonance. It is remarkable that it is only needed to guarantee the existence of a second $T$-periodic solution, while no such condition is necessary for the existence of the first one.

At a first sight, it could seem strange that the perturbing function $F$ is allowed to contain also friction terms. In the case when $\alpha=0$, this would not be possible, since a friction term would lead to dissipation of energy, thus eventually making the particle fall into the singularity. On the contrary, we will show that, when $\alpha \neq 0$, the rotating term $\alpha e^{i \omega t} /|x|^{\beta}$ forces the particle to maintain an almost constant rotation speed, and to remain at an almost constant distance from the singularity. Detailed information about the role of dissipative effects on Celestial Mechanics and the most important mechanisms of dissipation can be found in $[8,23]$, and the references therein.

\section{Proof of Theorem 1.}

After the change of variables

$$
s=\omega t, \quad z(s)=\left(\frac{\omega^{2}}{\alpha}\right)^{\frac{1}{\beta+1}} x(t),
$$

defining $G: \mathbb{R} \times\left(\mathbb{R}^{2} \backslash\{0\}\right) \times \mathbb{R}^{2} \times[0,1] \rightarrow \mathbb{R}^{2}$ as

$$
G(s, z, \zeta ; \varepsilon)=\left(\frac{1}{\alpha \omega^{2 \beta}}\right)^{\frac{1}{\beta+1}} F\left(\frac{s}{\omega},\left(\frac{\alpha}{\omega^{2}}\right)^{\frac{1}{\beta+1}} z, \omega\left(\frac{\alpha}{\omega^{2}}\right)^{\frac{1}{\beta+1}} \zeta ; \varepsilon\right)
$$

we get the equivalent equation

$$
\ddot{z}+c \frac{z}{|z|^{3}}=\frac{e^{i s}}{|z|^{\beta}}+\varepsilon G(s, z, \dot{z} ; \varepsilon),
$$

with

$$
c=\gamma\left(\frac{\omega^{2(2-\beta)}}{\alpha^{3}}\right)^{\frac{1}{\beta+1}} .
$$

Passing to polar coordinates

$$
z(s)=\rho(s) e^{i \theta(s)},
$$


equation (5) becomes

$$
\left(\ddot{\rho}-\rho \dot{\theta}^{2}+\frac{c}{\rho^{2}}\right)+i(2 \dot{\rho} \dot{\theta}+\rho \ddot{\theta})=\left(\frac{e^{i s}}{\rho^{\beta}}+\varepsilon G\left(s, \rho e^{i \theta},(\dot{\rho}+i \rho \dot{\theta}) e^{i \theta} ; \varepsilon\right)\right) e^{-i \theta},
$$

which is equivalent to the system

$$
\left\{\begin{array}{l}
\ddot{\rho}-\rho \dot{\theta}^{2}+\frac{c}{\rho^{2}}=\frac{\cos (s-\theta)}{\rho^{\beta}}+\varepsilon \widetilde{G}_{1}(s, \rho, \theta, \dot{\rho}, \dot{\theta} ; \varepsilon) \\
2 \dot{\rho} \dot{\theta}+\rho \ddot{\theta}=\frac{\sin (s-\theta)}{\rho^{\beta}}+\varepsilon \widetilde{G}_{2}(s, \rho, \theta, \dot{\rho}, \dot{\theta} ; \varepsilon),
\end{array}\right.
$$

where

$$
\begin{aligned}
& \widetilde{G}_{1}(s, \rho, \theta, \dot{\rho}, \dot{\theta} ; \varepsilon)=\Re\left(G\left(s, \rho e^{i \theta},(\dot{\rho}+i \rho \dot{\theta}) e^{i \theta} ; \varepsilon\right) e^{-i \theta}\right) \\
& \widetilde{G}_{2}(s, \rho, \theta, \dot{\rho}, \dot{\theta} ; \varepsilon)=\Im\left(G\left(s, \rho e^{i \theta},(\dot{\rho}+i \rho \dot{\theta}) e^{i \theta} ; \varepsilon\right) e^{-i \theta}\right)
\end{aligned}
$$

(For any complex number $w$, we denote by $\Re(w)$ and $\Im(w)$ its real and imaginary parts, respectively.) This change of variables is justified, since the solutions we are looking for never attain the singularity. Setting $\eta=\dot{\rho}, \varphi=s-\theta$, $v=\dot{\varphi}$ and defining

$$
\begin{aligned}
& \Gamma_{1}(s, \rho, \eta, \varphi, v ; \varepsilon)=\widetilde{G}_{1}(s, \rho, s-\varphi, \eta, 1-v ; \varepsilon), \\
& \Gamma_{2}(s, \rho, \eta, \varphi, v ; \varepsilon)=\widetilde{G}_{2}(s, \rho, s-\varphi, \eta, 1-v ; \varepsilon),
\end{aligned}
$$

we transform (7) into the first order system

$$
\left\{\begin{array}{l}
\dot{\rho}=\eta \\
\dot{\eta}=\rho(1-v)^{2}-\frac{c}{\rho^{2}}+\frac{\cos \varphi}{\rho^{\beta}}+\varepsilon \Gamma_{1}(s, \rho, \eta, \varphi, v ; \varepsilon) \\
\dot{\varphi}=v \\
\dot{v}=\frac{1}{\rho}\left(2 \eta(1-v)-\frac{\sin \varphi}{\rho^{\beta}}-\varepsilon \Gamma_{2}(s, \rho, \eta, \varphi, v ; \varepsilon)\right) .
\end{array}\right.
$$

Let us first consider the unperturbed equation, taking $\varepsilon=0$. There always is the equilibrium

$$
\mathcal{E}_{1}=\left(\rho_{1}, 0, \pi, 0\right)
$$

where $\rho_{1}$ satisfies

$$
\rho_{1}^{3}\left(1-\frac{1}{\rho_{1}^{\beta+1}}\right)=c .
$$

Notice that $\rho_{1}>1$. In this case, the orbit of the solution is tuned in such a way that the rotating force is always directed in the same direction as the 
gravitational force. For instance, in the particular case $\beta=2$, this equilibrium corresponds to the solution of $(1)$, with $\varepsilon=0$, given by

$$
x_{1}(t)=-\sqrt[3]{\frac{\gamma+\alpha}{\omega^{2}}} e^{i \omega t}
$$

Moreover, if $\beta \in[0,2[$, or $\beta=2$ and $\alpha<\gamma$, then there is a second equilibrium

$$
\mathcal{E}_{2}=\left(\rho_{2}, 0,0,0\right)
$$

where $\rho_{2}$ satisfies

$$
\rho_{2}^{3}\left(1+\frac{1}{\rho_{2}^{\beta+1}}\right)=c .
$$

In this case, the rotating force is always directed in the opposite direction with respect to the gravitational force. For instance, if $\beta=2$, we have

$$
x_{2}(t)=\sqrt[3]{\frac{\gamma-\alpha}{\omega^{2}}} e^{i \omega t}
$$

Let us first consider the linearization of system (8) at the equilibrium point $\mathcal{E}_{1}$, i.e., using (9),

$$
\dot{u}=\mathbb{A}_{1}\left(u-\mathcal{E}_{1}\right),
$$

with

$$
\mathbb{A}_{1}=\left(\begin{array}{cccc}
0 & 1 & 0 & 0 \\
3-\frac{2-\beta}{\rho_{1}^{\beta+1}} & 0 & 0 & -2 \rho_{1} \\
0 & 0 & 0 & 1 \\
0 & \frac{2}{\rho_{1}} & \frac{1}{\rho_{1}^{\beta+1}} & 0
\end{array}\right) .
$$

Let us evaluate the eigenvalues of $\mathbb{A}_{1}$, so to see whether they can or cannot be of the type $\lambda=i k$, with $k \in \mathbb{Z}$. We have

$$
\operatorname{det}\left(\mathbb{A}_{1}-\lambda I\right)=\lambda^{4}+\left(1-\frac{\beta-1}{\rho_{1}^{\beta+1}}\right) \lambda^{2}+\frac{1}{\rho_{1}^{\beta+1}}\left(3-\frac{2-\beta}{\rho_{1}^{\beta+1}}\right) .
$$

Setting $r_{1}=1 / \rho_{1}^{\beta+1}$ and $\lambda^{2}=\xi$, we have to solve the second order equation

$$
\xi^{2}+\left(1-(\beta-1) r_{1}\right) \xi+r_{1}\left(3-(2-\beta) r_{1}\right)=0
$$

whose discriminant is

$$
\Delta=\left(1-(\beta-1) r_{1}\right)^{2}-4 r_{1}\left(3-(2-\beta) r_{1}\right) \text {. }
$$


If $\Delta<0$, the solutions of (11) are nonreal, hence the eigenvalues of $\mathbb{A}_{1}$ are complex with nonzero real part. Otherwise, if $\Delta \geq 0$, the two solutions $\xi_{1}, \xi_{2}$ of (11) are real, and, since

$$
\xi_{1} \xi_{2}=r_{1}\left(3-(2-\beta) r_{1}\right)>0
$$

(recall that $\rho_{1}>1$, hence $r_{1}<1$ ), they have the same sign. Being

$$
\left.\xi_{1}+\xi_{2}=(\beta-1) r_{1}-1 \in\right]-2,0[,
$$

the only possibility of resonance is that $\xi_{i}=-1$, for some $i=1,2$. But, if this would happen, substituting in (11) would imply that $\beta<2$ and $r_{1}=$ $(2+\beta) /(2-\beta)$, which is impossible, since $r_{1}<1$. We thus conclude that the matrix $\mathbb{A}_{1}$ can never have eigenvalues of the type $\lambda=i k$, with $k \in \mathbb{Z}$.

Let us now assume that $\beta \in[0,2[$, or $\beta=2$ and $\alpha<\gamma$, and consider the linearization of system $(8)$ at the equilibrium point $\mathcal{E}_{2}$, i.e., using (10),

$$
\dot{u}=\mathbb{A}_{2}\left(u-\mathcal{E}_{2}\right)
$$

with

$$
\mathbb{A}_{2}=\left(\begin{array}{cccc}
0 & 1 & 0 & 0 \\
3+\frac{2-\beta}{\rho_{2}^{\beta+1}} & 0 & 0 & -2 \rho_{2} \\
0 & 0 & 0 & 1 \\
0 & \frac{2}{\rho_{2}} & -\frac{1}{\rho_{2}^{\beta+1}} & 0
\end{array}\right) .
$$

Let us evaluate the eigenvalues of $\mathbb{A}_{2}$. We have

$$
\operatorname{det}\left(\mathbb{A}_{2}-\lambda I\right)=\lambda^{4}+\left(1+\frac{\beta-1}{\rho_{2}^{\beta+1}}\right) \lambda^{2}-\frac{1}{\rho_{2}^{\beta+1}}\left(3+\frac{2-\beta}{\rho_{2}^{\beta+1}}\right) .
$$

Setting $r_{2}=1 / \rho_{2}^{\beta+1}$ and $\lambda^{2}=\xi$, we have to solve the second order equation

$$
\xi^{2}+\left(1+(\beta-1) r_{2}\right) \xi-r_{2}\left(3+(2-\beta) r_{2}\right)=0
$$

whose discriminant is

$$
\Delta=\left(1+(\beta-1) r_{2}\right)^{2}+4 r_{2}\left(3+(2-\beta) r_{2}\right)>0 .
$$

Hence its two solutions $\xi_{1}, \xi_{2}$ are real, and, since $\xi_{1} \xi_{2}<0$, one of them, say $\xi_{1}$, is negative, with

$$
\xi_{1}=-\frac{1}{2}\left(1+(\beta-1) r_{2}+\sqrt{\Delta}\right)
$$

A direct computation shows that $\xi_{1}<-1$. Moreover, for every $k \in \mathbb{Z}$ with $|k| \geq 2$, we have

$$
\xi_{1}=-k^{2} \quad \Longleftrightarrow \quad(2-\beta) r_{2}^{2}+\left((\beta-1) k^{2}+3\right) r_{2}+k^{2}\left(1-k^{2}\right)=0 .
$$

This second order equation has two real solutions, of which only one is positive. If we denote it by $r_{2}(k)$, it is explicitly given by $(3)$. 
By (6), since $r_{2}=1 / \rho_{2}^{\beta+1}$, we have the corresponding values of $c$, given by

$$
c(k)=\frac{r_{2}(k)+1}{r_{2}(k)^{\frac{3}{\beta+1}}} .
$$

It is possible to prove that $r_{2}(k)$ is strictly increasing with $k \geq 2$, and that $\lim _{k} r_{2}(k)=+\infty$. So, $c(k)$ is strictly decreasing, and

$$
\lim _{k \rightarrow+\infty} c(k)= \begin{cases}0 & \text { if } \beta \in[0,2[ \\ 1 & \text { if } \beta=2 .\end{cases}
$$

In particular, for every $k \geq 2$,

$$
c(k) \leq c(2)= \begin{cases}f(\beta) & \text { if } \beta \in[0,2[, \\ \frac{19}{12} & \text { if } \beta=2,\end{cases}
$$

with

$$
f(\beta)=\frac{5-6 \beta+\sqrt{16 \beta^{2}-56 \beta+97}}{\left(1-4 \beta+\sqrt{16 \beta^{2}-56 \beta+97}\right)^{\frac{3}{\beta+1}}}(4-2 \beta)^{\frac{2-\beta}{\beta+1}} .
$$

One can further prove that $f(\beta)$ is strictly increasing in $\beta \in[0,2[$, and that $\lim _{\beta \rightarrow 2^{-}} f(\beta)=\frac{19}{12}$ (see Figure 1$)$.

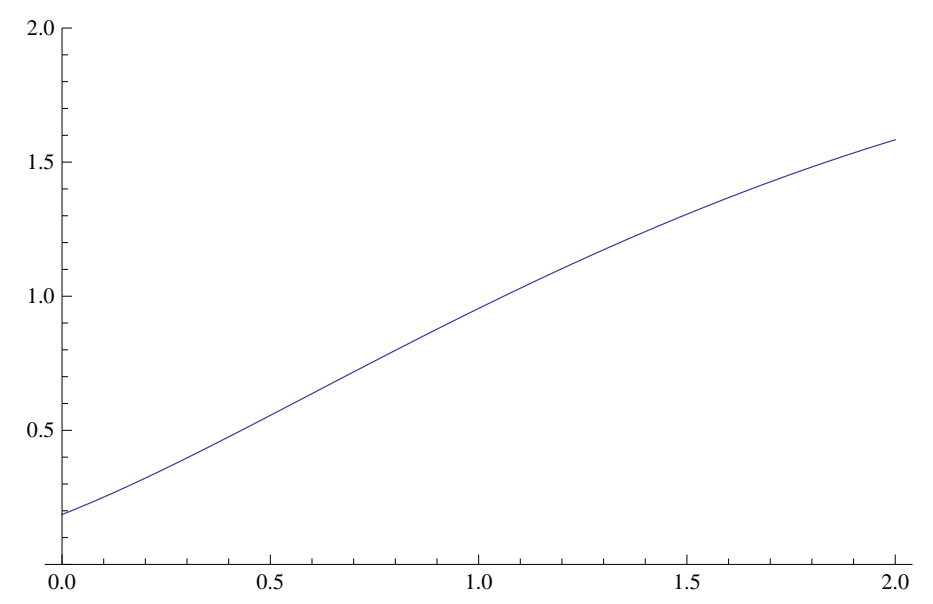

Figure 1: The function $f(\beta)$.

Now, we can conclude the proof. If $\varepsilon=0$, the linearized system at the equilibrium point $\mathcal{E}_{1}$ is always non resonant, i.e., the eigenvalues of $\mathbb{A}_{1}$ are never of the type $i k$, with $k \in \mathbb{Z}$. Therefore, since, for $j=1,2$, the functions $\Gamma_{j}(s, \rho, \eta, \varphi, v ; \varepsilon)$ are locally Lipschitz continuous in $(\rho, \eta, \varphi, v)$, a classical perturbation theorem applies (see, e.g., [10, Chapter 14, Theorem 1.1]), providing the existence of a $2 \pi$-periodic solution of (8), for $\varepsilon$ small enough. Correspondingly, by the changes of variables made above, we have a $T$-periodic solution of $(1)$. 
On the other hand, if $\beta \in[0,2[$, or $\beta=2$ and $\alpha<\gamma$, there is a second equilibrium point $\mathcal{E}_{2}$ for $\varepsilon=0$. The analysis of the linearized system at this equilibrium point shows the existence of some resonant values $c(k)$ for the constant $c$, given by (12), with $r_{2}(k)$ is defined as in (3). Since $c$ is defined as in (6), condition (2) (where $a(k)=r_{2}(k)$ ) is needed in order to avoid these resonant values. In this case, the above mentioned perturbation theorem applies, and one concludes analogously.

Since $c(k) \leq \frac{19}{12}$ for every $\beta \in[0,2]$ and every $k \in \mathbb{Z}$ with $|k| \geq 2$, if (4) is assumed, then (2) surely holds. The proof is thus concluded.

\section{References}

[1] A. Ambrosetti and V. Coti Zelati, Perturbation of Hamiltonian systems with Keplerian potentials. Math. Z. 201 (1989), 227-242.

[2] A. Ambrosetti and V. Coti Zelati, Periodic Solutions of Singular Lagrangian Systems. Birkhäuser, Boston, 1993.

[3] P. Amster, J. Haddad, R. Ortega and A. J. Ureña, Periodic motions in forced problems of Kepler type. NoDEA Nonlinear Differential Equations Appl., to appear.

[4] A. Bahri and P. H. Rabinowitz, A minimax method for a class of Hamiltonian systems with singular potentials. J. Funct. Anal. 82 (1989), 412-428.

[5] M. L. Bertotti, Forced oscillations of singular dynamical systems with an application to the restricted three body problem. J. Differential Equations 93 (1991), 102-141.

[6] H. Cabral and C. Vidal, Periodic solutions of symmetric perturbations of the Kepler problem. J. Differential Equations 163 (2000), 76-88.

[7] A. Capozzi, C. Greco and A. Salvatore, Lagrangian systems in the presence of singularities. Proc. Amer. Math. Soc. 102 (1988), 125-130.

[8] A. Celletti, L. Stefanelli, E. Lega and C. Froeschlé, Some results on the global dynamics of the regularized restricted three-body problem with dissipation, Celestial Mech. Dynam. Astronom. 109 (2011), 265-284.

[9] K.-Ch. Chen, Variational constructions for some satellite orbits in periodic gravitational force fields. Amer. J. Math. 132 (2010), 681-709.

[10] E. A. Coddington and N. Levinson, Theory of Differential Equations. McGraw-Hill, New York, 1955.

[11] B. Cordani, Perturbations of the Kepler problem in global coordinates. Celestial Mech. Dynam. Astronom. 77 (2000), 185-200. 
[12] M. Degiovanni, F. Giannoni and A. Marino, Dynamical systems with Newtonian type potentials. Atti Accad. Naz. Lincei Rend. Cl. Sci. Fis. Mat. Natur. (8) 81 (1987), 271-277.

[13] F. Diacu, E. Pérez-Chavela and M. Santoprete, The Kepler problem with anisotropic perturbations. J. Math. Phys. 46 (2005), 072701, 21 pp.

[14] A. H. Escalona-Buendía and E. Pérez-Chavela, Periodic orbits for anisotropic perturbations of the Kepler problem. Nonlinear Anal. 68 (2008), 591-601.

[15] A. Fonda and R. Toader, Periodic orbits of radially symmetric Keplerianlike systems: a topological degree approach. J. Differential Equations 244 (2008), 3235-3264.

[16] A. Fonda and R. Toader, Periodic solutions of radially symmetric perturbations of Newtonian systems. Proc. Amer. Math. Soc., online first.

[17] A. Fonda and A. J. Ureña, Periodic, subharmonic, and quasi-periodic oscillations under the action of a central force. Discrete Contin. Dyn. Syst. 29 (2011), 169-192.

[18] D. Franco and J. R. L. Webb, Collisionless orbits of singular and nonsingular dynamical systems. Discrete Contin. Dyn. Syst. 15 (2006), 747-757.

[19] D. Franco and P. J. Torres, Periodic solutions of singular systems without the strong force condition. Proc. Amer. Math. Soc. 136 (2008), 1229-1236.

[20] W. B. Gordon, Conservative dynamical systems involving strong forces. Trans. Amer. Math. Soc. 204 (1975), 113-135.

[21] M. Gutzwiller, The anisotropic Kepler problem in two dimensions. J. Math. Phys. 14 (1973), 139-152.

[22] T. Ito and K. Tanikawa, Trends in 20th century Celestial Mechanics. Publ. Natl. Astron. Observ. Japan 9 (2007), 55-112.

[23] A. Margheri, R. Ortega and C. Rebelo, Some analytical results about periodic orbits in the restricted three body problem with dissipation. Preprint available at www.ugr.es/ ecuadif/fuentenueva.htm.

[24] J. Moser and E. J. Zehnder, Notes on Dynamical Systems. Courant Lecture Notes, Amer. Math. Soc., 2005.

[25] T. Paul, On the status of perturbation theory. Math. Structures Comput. Sci., 17 (2007), 277-288.

[26] H. Poincaré, Les Méthodes Nouvelles de la Mécanique Céleste. GauthierVillars, Paris, 1892. 
[27] E. Serra and S. Terracini, Noncollision solutions to some singular minimization problems with Keplerian-like potentials. Nonlinear Anal. 22 (1994), 45-62.

[28] C. Siegel, J. Moser, Lectures on Celestial Mechanics. Springer, Berlin, 1971.

[29] P. J. Torres, Non-collision periodic solutions of forced dynamical systems with weak singularities. Discrete Contin. Dyn. Syst. 11 (2004), 693-698.

[30] C. Vidal, Periodic solutions for any planar symmetric perturbation of the Kepler problem. Celestial Mech. Dynam. Astronom. 80 (2001), 119-132.

Authors' addresses:

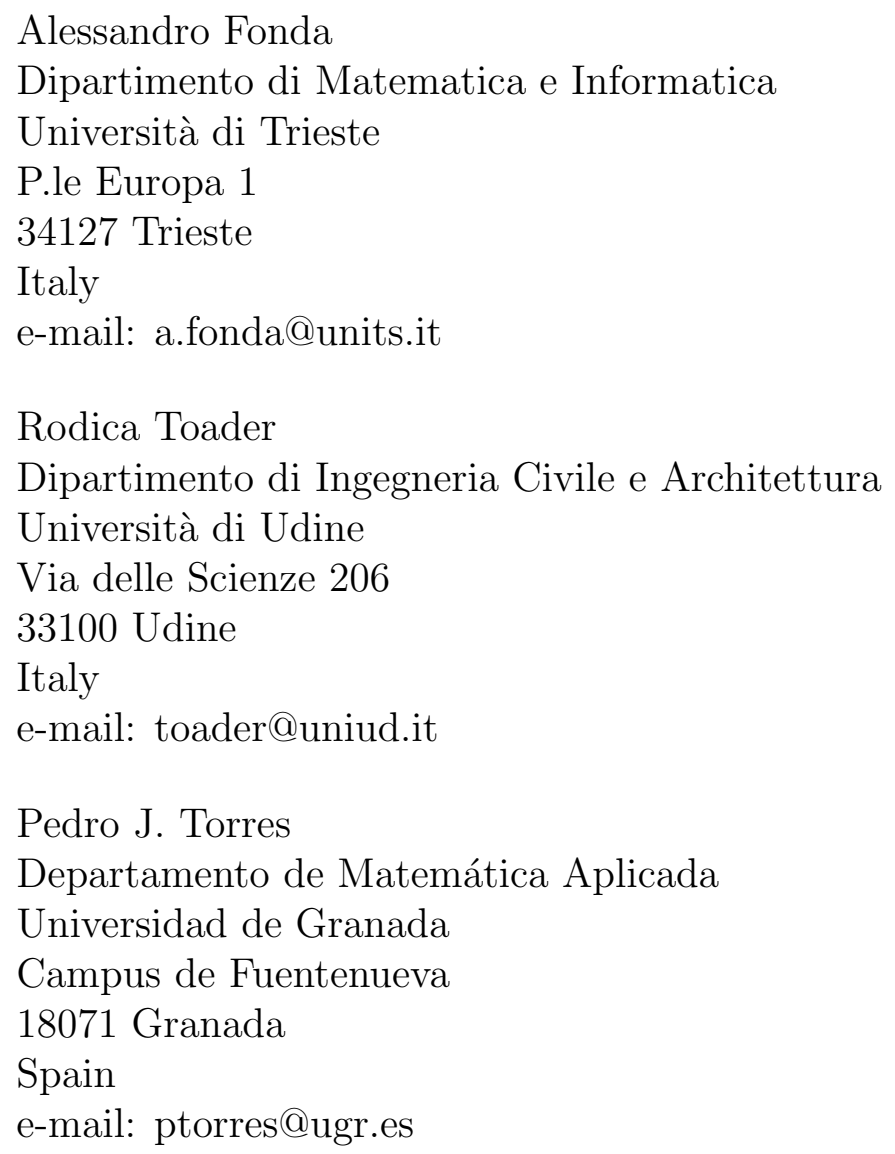

Keywords: periodic solutions; Kepler problem; perturbative methods. 